\section{Multiple Cranial Nerve Enhancement: A New MR Imaging Finding in Metachromatic Leukodystrophy}

Metachromatic leukodystrophy (MLD) is a set of several disorders caused by deficient lysosomal activity. This deficiency results in accumulation of a metachromatic lipid material, galactosylceramide sulfatide, leading to the breakdown of the myelin sheath in both central and peripheral nervous systems, initially sparing the subcortical " $U$ " fibers. ${ }^{1,2}$ Several MR imaging features of MLD have been described, but to the best of our knowledge, cranial nerve involvement demonstrated by MR imaging was never mentioned in this setting.

An undernourished 2-year-old girl was hospitalized as a result of uncontrollable vomiting and retarded neuropsychomotor development. Deep tendon reflexes were abolished in the 4 limbs, and the plantar reflex showed bilateral extension response. The routine laboratory tests, cranial nerve examination, and funduscopy were unremarkable. Electroneuromyography showed signs of predominantly sensory peripheral neuropathy in the lower limbs, with a demyelinating pattern that involved all 4 of the limbs, and an ultrastructural examination of the sural nerve was carried out, which showed abnormalities consistent with MLD. The disease was confirmed by a severe decrease of arylsulfatase A activity in leukocytes $(1.0 \mathrm{nmol} / \mathrm{h} / \mathrm{mg}$ of protein; reference range, $5-20 \mathrm{nmol} / \mathrm{h} / \mathrm{mg}$ ).

The baseline MR imaging scan showed bilateral and symmetrical involvement of the posterior periventricular white matter and splenium of the corpus callosum, sparing the U fibers. Gadolinium (Gd)enhanced T1-weighted images showed bilateral and symmetrical abnormal enhancement of several cranial nerves, including the optic, oculomotor, trigeminal, abducens, facial, and vestibulocochlear nerves. The follow-up scan (Fig 1) demonstrated diffuse and symmetrical demyelination in the periventricular white matter and centrum semiovale, with numerous hypointense linear structures in a radiating ("tigroid") pattern, with involvement of the internal capsules, corticospinal tracts, cerebral peduncles, and cerebellar white matter. There was no evidence of leptomeningeal or parenchymal enhancement. There was no evidence of nerve hypertrophy. There was a considerable worsening of the neurologic deficits characterized by spastic tetraplegia without any contact with the environment.

Cranial nerve Gd enhancement can be seen in a variety of entities, including neoplastic, infectious, demyelinating, and idiopathic diseases. $^{3-5}$ Among leukodystrophies, cranial nerve involvement has been documented in infantile Krabbe disease. ${ }^{6,7}$ Proposed mechanisms for the abnormal nerve root enhancement have included altered vascular permeability with breakdown of the blood-nerve bar-

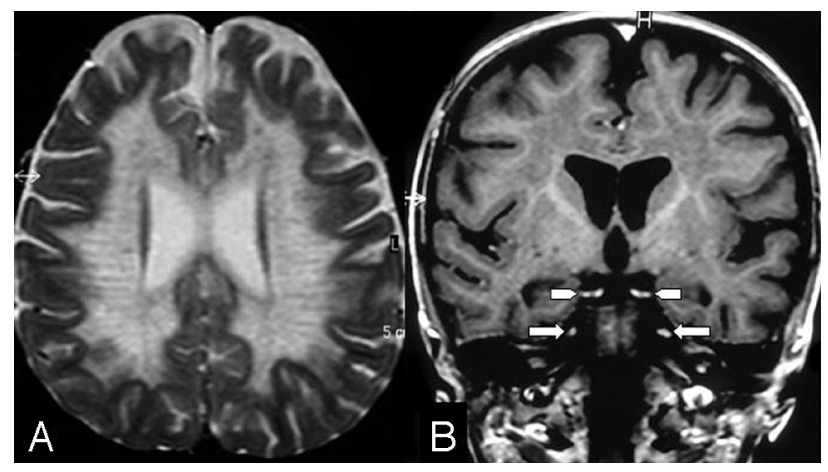

Fig 1. A, Axial T2-weighted image shows the white matter involvement with a tigroid pattern.

$B$, Coronal postcontrast T1-weighted image shows bilateral and symmetrical abnormal Gd enhancement of the oculomotor (arrowheads) and trigeminal nerves (arrows). rier as a result of perivascular inflammation or infiltration ${ }^{8}$ and enhancement in areas of active myelin breakdown. ${ }^{6}$

We presume that multiple cranial nerve enhancement in MLD is secondary to the accumulation of the metachromatic lipid material and to the disruption of the myelin sheath, similar to the process that affects peripheral nerves. A future investigation with a larger series is required to evaluate the usefulness of cranial nerve MR imaging analysis in suspected MLD patients.

\section{References}

1. Cheon JE, Kim IO, Hwang YS, et al. Leukodystrophy in children: a pictorial review of MR imaging features. Radiographics 2002;22:461-76

2. Faerber EN, Melvin J, Smergel EM. MRI appearances of metachromatic leukodystrophy. Pediatr Radiol 1999;29:669-72

3. Saremi F, Helmy M, Farzin S, et al. MRI of cranial nerve enhancement. AJR Am J Roentgenol 2005;185:1487-97

4. da Silva CJ, da Rocha AJ, Mendes MF, et al. Trigeminal involvement in multiple sclerosis: magnetic resonance imaging findings with clinical correlation in a series of patients. Mult Scler 2005;11:282-85

5. de Fátima Soares M, Braga FT, da Rocha AJ, et al. Optic nerve infiltration by acute lymphoblastic leukemia: MRI contribution. Pediatr Radiol 2005;35:799802

6. Bernal OG, Lenn N. Multiple cranial nerve enhancement in early infantile Krabbe's disease. Neurology 2000;54:2348-49

7. Jones BV, Barron TF, Towfighi J. Optic nerve enlargement in Krabbe's disease. AJNR Am J Neuroradiol 1999;20:1228-31

8. Vasconcellos E, Smith M. MRI nerve root enhancement in Krabbe disease. Pediatr Neurol 1998;19:151-52

Antonio C.M. Maia Jr Antônio J. da Rocha

Carlos J. da Silva

Section of Radiology

Sérgio Rosemberg

Division of Neuropediatrics

Department of Pediatrics

Santa Casa de Misericórdia de São Paulo

São Paulo, Brazil

DOI 10.3174/ajnr.A0526

\section{Congenital Glioependymal Cyst Presenting with Severe Proptosis}

We discuss a unique case of a congenital glioependymal cyst arising from the left middle cranial fossa with extension into the left orbit causing severe proptosis, a presentation that has not been reported previously. A female neonate at 38 weeks' gestational age was diagnosed with severe left proptosis on a prenatal sonography at 35 weeks' gestation. The sonogram was performed to determine the size of the infant due to a maternal history of macrosomia in a previous pregnancy. The infant's prenatal history was otherwise unremarkable. The patient was delivered by an uncomplicated cesarean birth.

Physical examination showed severe left proptosis with intact extraocular movements. The right eye and findings from the remaining physical examination were unremarkable. The results of chromosome studies were normal. Noncontrast enhanced CT demonstrated a cystic structure occupying the left middle cranial fossa and extending into the left orbit. MR imaging verified the CT findings and showed no associated brain abnormalities (Fig 1).

On the patient's second day of life, a cyst-to-peritoneum shunt was placed without complications. The left proptosis rapidly improved, and a sonogram showed only a small cyst on the fifth day of life. The patient was discharged but was returned on the 15th day of life because of recurrent left proptosis. After a failed shunt revision, a left temporal craniotomy was performed with removal of the lateral wall of the cyst and fenestration of the cyst to the subarachnoid space. 

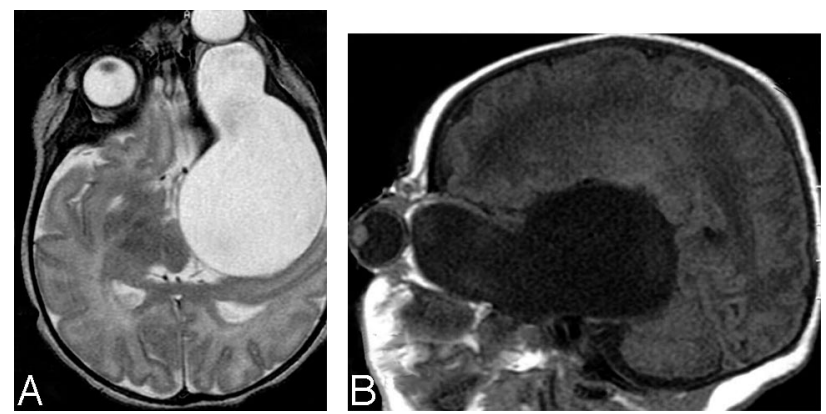

Fig 1. Noncontrast MR imaging of axial $\mathrm{T} 2(A)$ and sagittal $\mathrm{T} 1(B)$ images show the cystic structure after CSF signal intensity on all sequences. Note proptotic left globe.

No brain parenchymal abnormalities were identified. Since that time, the child has had an uneventful clinical course, and a noncontrast CT 1 year later showed that only a small cyst remained.

Upon gross examination, the pathologic specimen contained multiple fragments of fibrous tissue in loose aggregates (not photographed). Microscopic examination revealed that the specimen consisted of a thick layer of glial/connective tissue lined by a single layer of cuboidal or columnar epithelium with cilia. In certain areas, papillary structures resembling the choroid plexus were identified. No goblet cells were seen in the specimen. The epithelium was diffusely positive for S-100 protein and glial fibrillary acidic protein, and focally positive for cytokeratin in the areas resembling the choroid plexus. The epithelial cells were negative for epithelial membrane antigen and carcinoembryonic antigen. Focal acute inflammation was noted, consistent with previous surgical intervention. The histologic and immunophenotypic findings were most consistent with a glioependymal cyst.
Based on the imaging features, the differential diagnoses included a glioependymal cyst, ependymal cyst, arachnoid cyst, enterogenous cyst, dermoid cyst, encephalocele, and congenital dysplasia of the sphenoid wing in neurofibromatosis type I. The diagnosis of a glioependymal cyst requires correlation of clinical, radiologic, and histologic findings. Glioependymal cysts, also known as neuroepithelial cysts, are thought to arise from ectopic rests of primitive neuroglial tissue and therefore can arise anywhere in the neuraxis. ${ }^{1}$ On imaging, they appear as nonenhancing CSF containing thin-walled cysts. ${ }^{2,3}$ Our case is unique because of the unusual clinical presentation of severe proptosis. To our knowledge, neither this presentation nor the imaging appearance has been reported previously in a neonate with a glioependymal cyst.

\section{References}

1. Osborn AG, Preece MT. Intracranial cysts: radiologic-pathologic correlation and imaging approach. Radiology 2006;239:650-64

2. Shields JA, Shields CL. Orbital cysts of childhood-classification, clinical features, and management. Surv Ophthalmol 2004;49:281-99

3. Guermazi A, Miaux Y, Majoulet JF, et al. Imaging findings of central nervous system neuroepithelial cysts. Eur Radiol 1998;8:618-23

Ruby E. Obaldo

Department of Radiology

The University of Kansas Medical Center

Kansas City, Kan

Lei Shao

Department of Pathology

Lisa $\mathrm{H}$. Lowe

Department of Radiology

Children's Mercy Hospital and Clinics and The University of Missouri-Kansas City

Kansas City, Mo

DOI 10.3174/ajnr.A0533 